\title{
NOTIONS OF NORM-ATTAINABILITY AND ORTHOGONALITY IN BANACH SPACES
}

\author{
W. L. OTAE, N. B. OKELO*, O. ONGATI \\ Department of Pure and Applied Mathematics, \\ Jaramogi Oginga Odinga University of Science and Technology, \\ Box 210-40601, Bondo-Kenya \\ ${ }^{*}$ Corresponding author: bnyaare@yahoo.com
}

Received Apr. 20, 2021

\begin{abstract}
New notions of norm-attainability and orthogonality are given in this paper. We first consider Range-Kernel orthogonality in norm-attainable classes then we characterize orthogonality conditions for Jordan elementary operators in a Banach space setting. 2010 Mathematics Subject Classification. 47B47; 47A30.
\end{abstract}

Key words and phrases. orthogonality; elementary operator; norm-attainablility; Banach space.

\section{INTRODUCTION}

Studies in normed spaces have been carried out with very interesting results obtained as shown in [2], [4] and [14]. Several properties of operators in these spaces have been studied including norms, orthogonality, spectra, among others in Hilbert spaces and Banach spaces in general [24]. Regarding norm-attainable classes, a lot has been done in terms of structural properties [16]- [23]. Elementary operators have also been of considerable attention on many aspects particularly on their orthogonality (see [3] and the references therein). Let $H$ be an infinite dimensional complex Hilbert space and $B(H)$ the algebra of all bounded linear operators on $H$. We say $T \in B(H)$ is said to be norm-attainable if there exists a unit vector $x_{0} \in H$ such that $\left\|T x_{0}\right\|=\|T\|$. We denote by $N A(H)$ the class of all norm-attainable operators on $H$. Benitez [1] gave a detailed description of several types of orthogonality which have been studied in real normed spaces namely: Robert's orthogonality, Birkhoff's orthogonality,

DOI: $10.28924 /$ APJM/8-16

(92021 Asia Pacific Journal of Mathematics 
Orthogonality in the sense of James, Isosceles, Pythagoras, Carlsson, Diminnie, Area among others as described in [12]. For $x \in \mathcal{M}$ and $y \in \mathcal{N}$, where $\mathcal{M}$ and $\mathcal{N}$ are subspaces of $E$ which is a normed linear space, we have: Roberts- $\|x-\lambda y\|=\|x+\lambda y\|, \forall, \lambda \in \mathbb{R} ;$ Birkhoff$\|x+\lambda y\| \geq\|x\| ;$ Isosceles- $\|x-y\|=\|x+y\| ;$ Pythagorean- $\|x-y\|^{2}=\|x\|^{2}+\|y\|^{2} ;$ a-Pythagorean$\|x-a y\|^{2}=\|x\|^{2}+a^{2}\|y\|^{2}, a \neq 0 ;$ Diminnie-sup $\left\{f(x) g(y)-f(y) g(x): f, g \in S^{\prime}\right\}=\|x\|\|y\|$ where $S^{\prime}$ denotes the unit sphere of the topological dual of $E$; Area- $\|x\|\|y\|=0$ or they are linearly independent and such that $x,-x, y,-y$ divide the unit ball of their own plane (identified by $\mathbb{R}^{2}$ ) in four equal areas [24]. For characterizations of elementary operators, we consider Banach algebras and norm-attainable classes. Let $\mathcal{A}$ be a normed space and let $T_{A, B}: \mathcal{A} \rightarrow \mathcal{A} . T$ is called an elementary operator if it has the following representation: $T(X)=\sum_{i=1}^{n} A_{i} X B_{i}, \forall X \in \mathcal{A}$, where $A_{i}, B_{i}$ are fixed in $\mathcal{A}$. Let $\mathcal{A}=B(H)$. For $A, B \in B(H)$ we define specific elementary operators as follows [15]: The left multiplication operator $L_{A}: B(H) \rightarrow B(H)$ by $L_{A}(X)=A X, \forall X \in B(H)$; the right multiplication operator $R_{B}: B(H) \rightarrow B(H)$ by $R_{B}(X)=X B, \forall X \in B(H)$; the generalized derivation (implemented by $A, B$ ) by $\delta_{A, B}=L_{A}-R_{B}$; the basic elementary operator(implemented by $A, B$ ) by $M_{A, B}(X)=A X B, \forall X \in B(H)$; the Jordan elementary operator(implemented by $A, B$ ) by $\mathcal{U}_{A, B}(X)=A X B+B X A, \forall X \in B(H)$.

In this paper, we characterize the orthogonality of the range and the kernel of several types of important elementary operators in normed spaces. Let $X \in B(H)$ be a compact operator, and let $s_{1}(X) \geq s_{2}(X) \geq . . \geq 0$ denote the eigenvalues of $|X|=(X * X)^{\frac{1}{2}}$ arranged in their decreasing order. Considering the normed classes, we call $C_{p:_{1 \leq p<\infty}}(H)$ (simply denoted by $\left.C_{p}(H)\right)$, the Von Neumann-Schatten $p$-class, if $\|X\|=\left[\sum_{i=1}^{\infty} S_{i}(X)^{p}\right]^{\frac{1}{p}}=\operatorname{tr}\left(|X|^{p}\right)^{\frac{1}{p}}<+\infty$, where $t r$ denotes the trace functional. Hence, $C_{1}(H)$ is the trace class, $C_{2}(H)$ is the HilbertSchmidt class and the case $p=\infty$, corresponds to the class of compact operators $C_{\infty}(H)$ equipped with the norm $\|X\|_{\infty}=S_{1}(X)$. For more details, the reader is referred to [8]- [23] and the references therein.

\section{Preliminaries}

In this section, we give the basic concepts which are useful in the sequel. We begin with the following definition.

Definition 2.1. Let $W$ be a complex normed space, then for any elements $x, y \in W$, we say that $x$ is orthogonal to $y$, noted by $x \perp y$, if and only if for all $\alpha, \beta \in \mathbb{C}$ there holds $\|\alpha y+\beta x\| \geq\|\beta x\|$. 
Definition 2.2. Let $W$ be a complex Banach space. If $P$ and $Q$ are linear subspaces in $W$, we say that $P$ is orthogonal to $Q$, noted by $P \perp Q$, if $\|x+y\| \geq\|x\|$ for all $x \in P$ and all $y \in Q$. If $P=x$, we simply write $x \perp Q$.

Remark 2.3. We note that the orthogonality in the definition above is not symmetric [5] and if $W$ is a Hilbert space with its inner product then it follows from [7] that $\langle x, y\rangle=0$ which means that Birkhoff-James orthogonality generalizes the usual sense of orthogonality in a Hilbert space [6].

Definition 2.4. Let $F: X \rightarrow Y$ and $J: Y \rightarrow Z$ be operators between norm-attainable classes. We say $J$ is called orthogonal to $F$ if $s \in \operatorname{Ker} T \Rightarrow\|s+E(x)\| \geq\|s\|, \forall x \in X$. Moreover, if $F=J$, we shall say that $F$ is orthogonal.

Remark 2.5. The authors in [20] proved that if $A$ and $B$ are normal operators then for all $X, S \in$ $B(H), S \in \operatorname{ker} \delta_{A, B} \Rightarrow\left\|\delta_{A, B}(X)+S\right\|=\|S\|$, where the ker $\delta_{A, B}$ denotes the kernel of $\delta_{A, B}$. This means that the kernel of $\delta_{A, B}$ is orthogonal to its range. This result has been generalized in different directions, to non-normal operators [25], to $C_{p}(H)$, and to some elementary operators [6].

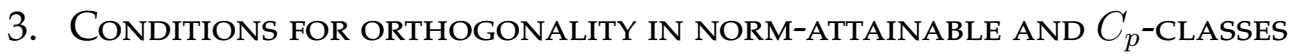

Let $X$ be a normed linear space over the field $\mathbb{K}$ and $X^{\dagger}$ its topological dual. For all $x \in X$, we have $D(X)=\left\{\varphi \in X^{\dagger}: \varphi(x)=\|x\|^{2} ;\|\varphi\|=\|x\|\right\}$ called the duality mapping. The HahnBanach's theorem [22] ensures that there exists always at least one support functional (a support functional $\varphi$ at $x \in X$ is a norm-one linear functional in $X^{\dagger}$ such that $(\varphi(x)=\|x\|)$ at each vector $x \in X$ ) and therefore $D(x)$ is non-empty for every $x \in X$. Moreover, it is well known that $D(x)$ is convex and weak*-compact subset of $X^{\dagger}$. Hence, $D$ is not linear in general but it is homogeneous, that is, for all $\alpha \in \mathbb{R}, D(\alpha x)=\alpha D(x)$.

Proposition 3.1. Let $K$ be a norm-attainable subclass of a norm-attainable set $X$ and $x \notin K$, then $x \perp K$ if and only if there exists $\tilde{\varphi} \in D(x)$ such that $K \subseteq k e r \tilde{\varphi}$.

Proof. Since $K$ is a norm-attainable class, let $\varphi \in D(x)$ be such that $\langle\varphi, y\rangle=0$, for all $y \in K$. Then $\varphi(x+y)=\varphi(x)=\varphi\|x\|^{2}$ and $\|x\|^{2}=\|\varphi\|\|x+y\|=\|x\|\|x+y\|$, that is, $\|x\|=\|x+y\|$, for all $y \in K$. Hence, $x \perp K$.

Conversely, let $x \notin K$ such that $x \perp K$. Then, for all $y \in K, x$ and $y$ are linearly independent vectors. Let $L$ be the closed norm-attainable subclass spanned by $K$ and $x, L=[K, x]$, and define the function $\varphi$ on $L$ by $\varphi(\alpha x+\beta y)=\alpha\|x\|^{2}$, for all $y \in K$ and all $\alpha, \beta \in \mathbb{C}$. Clearly, $\varphi$ is linear (by the assumption that $K$ is a linear subset of $X$ ). To prove the continuity of $\varphi$, 
let $z \in L$, then $z=\alpha x+\beta y$ and $\varphi(z)=\alpha\|x\|^{2}$. By the definition of $\varphi$ and the assumption that $x \perp K$, we derive that $\|z\|=\|\alpha x\|$. If $\alpha \neq 0$, it is easy to see from known inequalities that $|\varphi(z)|=|\alpha|\|x\|^{2} \cdot \frac{\|z\|}{\|z\|} \leq \frac{|\alpha|\|x\|^{2}}{\|\alpha x\|}\|z\|=\|x\|\|z\|$. If $\alpha=0$, then $|\varphi(z)|=|\varphi(\beta y)|=0$. Hence, $|\varphi(z)| \leq\|x\|\|z\|$, for all $z \in L$. Therefore, $\varphi$ is continuous on $L$ and $\|\varphi\|=\|x\|$. By Hahn-Banach theorem there is a continuous linear functional $\tilde{\varphi}$ on $X$ such that $\tilde{\varphi}_{\mid L}=\varphi$ and $\|\tilde{\varphi}\|=\|\varphi\|$, where $\tilde{\varphi}_{\mid L}$ is the restriction of $\varphi$ on $L$. It follows, by the definition of $\varphi$ and $\tilde{\varphi}_{\mid L}=\varphi$ that $K \subseteq \operatorname{ker} \tilde{\varphi}$ and $\tilde{\varphi} \in D(x)$.

Theorem 3.2. Let $K$ be a norm-attainable subclass of a norm-attainable set $X$. For all $x, y \in X x \perp y$ if and only if there exists $\varphi \in D(x)$ such that $\varphi(y)=0$. Moreover, for all $x \in X$ and for all $\varphi \in D(x)$, $x \perp \operatorname{ker} \varphi$.

Proof. From Proposition 3.1 and an analogous computation from [25], the proof is clear.

Remark 3.3. We can consider general normed spaces as follows. Let $K$ be a nonempty subset of a Banach space $X$ and $T \in N A(X)$, we denote the duality adjoint of $T$ by $T^{\dagger}$ and set $K^{\perp r}=\{x \in X$ : $x \perp y ; \forall y \in K\}$. It is clear that if $\left\{x_{n}\right\}_{n}$ is a sequence in a subset $K$ converging to $y$ and $x \perp x_{n}$, for all $n$, then $x \perp y$. Hence, $x \perp K \rightarrow x \perp \bar{K}$.

Proposition 3.4. Let $X$ be a norm-attainable subclass of $N A(H)$

(1). If $K$ and $L$ are closed subclasses of $X$ and $K \oplus L$

(2). Let $T \in N A(H)$ and $s \in X$. Then

(i). $s \perp \operatorname{ran} T \Leftrightarrow \exists \varphi \in D(s)$ such that $\varphi \in \operatorname{ker} T^{\dagger}$.

(ii). If $T$ is orthogonal, then $\operatorname{ker} T \oplus \overline{\operatorname{ran}(T)}$ is a closed subclass of $X$.

Proof. (1). Let $z \in X: z=\lim _{n}\left(x_{n}+y_{n}\right)$ and $z_{n}=\left(x_{n}+y_{n}\right)$; for all $n \geq 1$, where $x_{n} \in$ $K$ and $y_{n} \in L$. From $K \in L^{\perp r}$, we obtain,

$\left\|z_{n}-z_{n+p}\right\|=k\left\|y_{n}-y_{n+p}+x_{n}-x_{n+p}\right\|=\left\|x_{n}-x_{n+p}\right\|$, for all $n, p$.

So, $\left\{x_{n}\right\}_{n}$ is a Cauchy sequence, hence $\lim _{n} x_{n} \in K$. Setting $x=\lim _{n} x_{n}$, we get $\lim _{n} y_{n}=$ $z-x \in L$ and therefore, $z \in K \oplus L$.

(2) (i). By Proposition 3.1, $s \perp \operatorname{ran} T \Leftrightarrow \exists \varphi D(s), \operatorname{ran}(T) \subseteq \operatorname{ker} \varphi$ that is $\varphi(T x)=\left(T^{\dagger} \varphi\right) x=0$, for all $x \in X$. Hence $s \perp \operatorname{ran} T \Leftrightarrow \exists \varphi \in D(s): \varphi \in \operatorname{Ker} T^{\dagger}$.

$($ ii $)$. It is a direct consequence of the assertions (1) and $(2)(i)$.

Remark 3.5. Let $f: X \rightarrow X$ be a map on $X$, not necessarily linear or additive, and $F_{f}: X \rightarrow \mathbb{R}^{+}$ be a map defined by $F_{f}(x)=\|f(x)\|, \forall x \in X$. We say that $F_{f}$ has a global minima at a $\in X$ if 
$\|f(a)\|=\|f(x)\|$, for all $x \in X$. As an application of the previous results, the following theorem gives us a necessary and sufficient conditions in term of Birkhoff-James orthogonality for minimizing the $\operatorname{map} F_{f}$.

Lemma 3.6. Let $T$ and $f: X \rightarrow X$ be norm-attainable maps and $a \in X$. Suppose that the relation $f(x)+T(y)=f(y)+T(x)$ holds for all $x, y \in X$, if $f(a) \perp T(x), \forall x \in X$ then the map $F_{f}$ has $a$ global minima at a. Moreover, if we suppose that $T$ is linear and $f(x)=T(x)+f(0), \forall x \in X$, then $F_{f}$ has a global minima at $a$ if and only if $f(a) \perp \operatorname{ranT}$ if and only if there is $\varphi \in D(f(a))$ such that $\varphi \in \operatorname{ker} T^{\dagger}$, where $T^{\dagger}$ is the duality map of $T$. Lastly, if $f(a)$ is a smooth point, then the existence of $\varphi$ is unique.

Proof. It follows from Theorem 3.2, that for all $x \in X, f(a) \perp T(x) \Leftrightarrow \exists \varphi \in X^{\dagger}: \varphi(f(a))=$ $\|f(a)\|_{2}=\|\varphi\|^{2}$ and $\varphi(T(x))=0$. Then by the relation defined in (i), we get $\varphi(f(a))=$ $\varphi(f(a)+T(x))=\varphi(f(x)+T(a))=\varphi(f(x))$, so that $\|\varphi(f(a))\|=\|f(a)\|\|\varphi\| \leq\|\varphi\|\|f(x)\|$. Hence, $\|f(a)\| \leq\|f(x)\|$, for all $x \in X$. Since the maps $f, T$ satisfy the relation cited in (i), the sufficient condition follows from the first part of the proof. By linearity of $T$, we get $f(a)+\lambda T(x)=f(a+\lambda x)$, for all $x \in X, \lambda \in \mathbb{C}$. Hence, $F_{f}$ has a global minima at a implies $\|f(a)+\lambda g(x)\| \geq\|f(a+\lambda x)\|=\|f(a)\|$. The other equivalence follows immediately. To complete the proof, if $f(a)$ is a smooth point, then $f(a)$ has only one functional support and therefore $D(f(a))$ has one element.

Lemma 3.7. If $\mathcal{J}$ is a separable ideal of norm-attainable operators in $N A(H)$ equipped with unitary invariant norm, then its dual $\mathcal{I}$ is isometrically isomorphic to an ideal of compact operator $\mathcal{Q}$ not necessarily separable as described in the following i.e. $\phi: \mathcal{Q} \rightarrow \mathcal{J}^{\dagger}, R \mapsto \phi_{R}(X)=\operatorname{tr}(X R)$.

Proof. Following the argument in Lemma 3.6, the proof is trivial.

Theorem 3.8. Let $A \in C_{p}(H), T \in B\left(C_{p}(H)\right)$ and $f, F_{f}$ are defined as in Lemma 3.6, where $f(A)$ is given by its polar decomposition $f(A)=u|f(A)|$. If $A \in C_{p}(H)$, then $F_{f}$ has a global minimizer at $A$ if and only if $f(A) \perp \operatorname{ranT}$ if and only if $|f(A)|^{p-1} u^{*} \in \operatorname{ker} T^{\dagger}$. Moreover, if $A \in C_{1}(H)$ and $f(A)$ is a smooth point, then $F_{f}$ has a global minimizer at $A$ if and only if $f(A) \perp$ ranT if and only if $u^{*} \in \operatorname{ker} T^{\dagger}$ when $f(A)$ is injective (or $u \in \operatorname{ker} T^{\dagger}$ when $f(A)^{*}$ is injective).

Proof. From Lemma 3.6, we have that $F_{f}$ has a global minimizer at $A$ if and only if there exists $\varphi \in D(f(A))$ such that $\varphi \in k e r T^{\dagger}$. If $A \in C_{p}(H)$, then by the properties of the isomorphism, it follows that $\varphi \in C_{p}(H)^{\dagger}$ if and only if there exists $R \in C_{p}(H)$ such that $\phi_{R}=\varphi,\|\varphi\|=\|R\|$ and 
$\varphi(X)=\operatorname{tr} R X$, for all $X \in C_{p}(H)$. Hence, the smoothness of $C_{p}(H), F_{f}$ has a global minimizer at $A$ if and only if there is a unique operator $R$ such that $\varphi(f(A))=\operatorname{tr}(f(A) R)=\|f(A)\|_{p}^{2}=$ $\|R\|_{q}^{2}$ and $\operatorname{tr}\left(T^{\dagger}(R) X\right)=0$, for all $X \in C_{p}(H)$. To complete the proof, it is well known that $C_{1}(H)$ is neither reflexive, nor smooth and its dual $C_{1}(H)^{\dagger}$ is isometrically isomorphic to $N A(H)$. This isomorphism is given by $\phi \in N A(H)$ if and only if $C_{1}(H)^{\dagger}$ contains $R \mapsto \phi_{R}$ such that $\phi_{R}(X)=\operatorname{tr}(X R)$ so we have that $\varphi \in C_{1}(c H)^{\dagger} \Leftrightarrow \exists R \in N A(H): \phi_{R}=\varphi,\|\varphi\|=\|R\|$ and $\varphi(X)=\operatorname{tr} R X \forall X \in C_{1}(H)$, so that if $f(A)$ is a smooth point then $F_{f}$ has a global minimizer at $A$ if and only if there is a unique operator $R$ such that $\varphi(f(A))=\operatorname{tr}(f(A) R)=\|f(A)\|_{1}^{2}=\|R\|^{2}$ and $\operatorname{tr}\left(T^{\dagger}(R) X\right)=0, \forall X \in C_{1}(H)$. Since $f(A)$ is smooth then by [17], either $f(A)$ or $f(A)^{*}$ is injective, thus either $u$ or $u^{*}$ is an isometry i.e., $u u^{*}=I$ or $u^{*} u=I$. So it suffices to take $R=\|f(A)\|_{1} u^{*}$ or $R=\|f(A)\|_{1} u$ which is the unique operator required in both cases. So, $f(A)$ or $f(A)^{*}$ is injective.

Proposition 3.9. Let $K$ be a closed subclass of a norm-attainable class $X$. If $X$ is separable and $K^{\perp r}=0$, then $K=X$.

Proof. If $K \neq X$, then there exists $\varphi \in X^{\dagger}: K \subseteq \operatorname{ker} \varphi$. Since $D(\varphi)$ is not empty, then there is $f \in X^{\dagger \dagger}$ with $f(\varphi)=\|\varphi\|^{2}=\|f\|^{2}$. Let $J$ be the natural injection between $X$ and $X^{\dagger \dagger}$ i.e. $J: X \rightarrow X^{\dagger \dagger}, \forall x \in X, \forall \psi \in X^{\dagger}$, such that $J(x) \psi=\psi(x),\|J(x)\|=\|x\|$. So, by the separability of $X, J$ is a bijection and, then there is $0 \neq x \in X$ such that $J(x)=f$. Hence, $\varphi(x)=\|x\|^{2}=$ $\|\varphi\|^{2}$. Thus, $\varphi \in D(x)$ and by application of Proposition 3.1, we get $0 \neq x \in K^{\perp r}=0$, a contradiction.

Proposition 3.10. Let $X$ be a separable, smooth and strictly convex norm-attainable class and $T \in$ $N A(X)$. If $T^{\dagger}$ is orthogonal, then $\forall s \in X, s \perp \operatorname{ran} T \Rightarrow s \in \operatorname{Ker} T$.

Proof. Let $s \in X$ such that $\perp \operatorname{ranT}$. Then by [17] and the smoothness of $X$, there is a unique $\varphi_{s} \in D(s)$ such that $\varphi_{s} \in k e r T^{\dagger}$. Again, by assumptions of the proposition and arguments in [15], there is $\psi_{\varphi_{s}} \in D\left(\varphi_{s}\right)$ such that $\psi_{\varphi_{s}} \in \operatorname{ker} T^{\dagger \dagger}$. Let $J$ be the natural injection between $X$ and $X^{*}$ as defined in [10]. We see that $J(s) \varphi_{s}=\left\|\varphi_{s} k\right\|$ and $\|J(s)\|=\left\|\varphi_{s}\right\|$, which means that $J(s) \in D\left(\varphi_{s}\right)$. By the separability of $X, J$ is a bijection. Hence, there is $c \in X$ such that $\psi_{\varphi_{s}}=J(c)$ and $\|J(c)\|=\|c\|=\|\varphi\|, J(c) \varphi_{s}=\varphi_{s}(c)=\psi_{\varphi_{s}}\left(\varphi_{s}\right)=\left\|\varphi_{s}\right\|^{2}$. Then $\varphi_{s} \in D(s) \bigcap D(c)$ and since $X$ is strictly convex, we get $c=s$. Thus, $J(c)=J(S) \in \operatorname{ker} T^{\dagger \dagger}$. Therefore, it immediately follows that $\left(T^{\dagger \dagger} J(s)\right) \varphi=J(s)\left(T^{\dagger}\right)=\left(T^{\dagger} \varphi\right) s=\varphi(T s)=0$, for all $\varphi \in X^{\dagger}$. That is $s \in k e r T$. 
In the next result we consider general Banach spaces. If $X$ is a reflexive separable Banach space and $T^{\dagger}$ is orthogonal then the implication of orthogonality holds with respect to a suitable norm in $X$. Indeed, if $X$ is separable, then there is an equivalent norm which is smooth and strictly convex in $X$. This is seen in the next theorem.

Theorem 3.11. Let $X$ be a reflexive, smooth and strictly convex Banach space and $T \in B(X)$. If $T$ and $\dagger$ are orthogonal. Then $\forall s \in X: s \perp \operatorname{ran} T \Leftrightarrow s \in \operatorname{ker} T$, where $X=\operatorname{ker} T \oplus \overline{\operatorname{ran}(T)}$.

Proof. If $T$ is orthogonal then, by Definition 2.2, it follows that $\forall s \in X$ such that $s \perp \operatorname{ranT}$ it implies that $s \in k e r T$ and the reverse implication follows by Proposition 3.1. Let us prove the decomposition. Let $y \in X$ such that $y \in(\operatorname{ker} T \oplus \operatorname{ran}(T))^{\perp r}$, then there is $\varphi_{y} \in D(y)$ such that $\varphi_{y}(s \oplus T X)=0$, for all $s \in \operatorname{ker} T$ and all $x \in X$. For $s=0$, it follows, by [22], that $Y \perp \operatorname{ranT}$, and by [23], $\operatorname{ran} T \subseteq \operatorname{ker} T$. So, we can choose $x=0$ and $s=y$, such that this yields $\varphi_{y}(y)=0$. This means $y \perp y$, and hence $y=0$. Finally, the decomposition follows immediately.

\section{ORTHOGONALITY CONDITIONS FOR ELEMENTARY OPERATORS}

In this section, we consider the important case, when the operator $T$, cited in the previous section, is replaced by the elementary operators defined as follows: $E(X)=\sum X_{i=1}^{n} A_{i} X B_{i}$ on $C_{p}(H)$ where $A=\left(A_{1}, A_{2}, \ldots, A_{n}\right)$ and $B=\left(B_{1}, B_{2}, \ldots, B_{n}\right)$ are $n$-tuples in $(N A(H))_{n}$. The duality adjoint of $E$ on $C_{p}(H)$ has the form $E^{\dagger}(X)=\sum_{i=1}^{n} B_{i} X A_{i}$. Indeed, let $X \in C_{p}(H)$ and $R \in B(H)\left(\right.$ or $R \in C_{q}(H)$ if $X \in C_{p}(H)$, where we have $\frac{1}{p}+\frac{1}{q}=1$ and that $1<p, q<\infty$ then the following form suffices, that is,

$\phi_{R}(E(X))=\operatorname{tr}\left(\sum_{i=1}^{n} A_{i} X B_{i} R\right)=\operatorname{tr}\left(X \sum_{i=1}^{n} A_{i} R B_{i}\right)=\operatorname{tr}\left(X E^{\dagger}(R)\right)=\phi_{E^{\dagger}(R)}(X)$. We denote the formal adjoint of $E$ by $\tilde{E}=\sum_{i=1}^{n} A_{i}^{*} X B_{i}^{*}$ where $\left(A_{1}^{*}, A_{2}^{*}, \ldots, A_{n}^{*}\right)$ and $\left(B_{1}^{*}, B_{2}^{*}, \ldots, B_{n}^{*}\right)$ are $n$-tuples of operators in $(B(H))^{n}$.

Proposition 4.1. Let $H, K$ be Hilbert spaces, $A \in N A(H), B \in N A(K)$ and $E \in B(N A(K, H))$ such that $E(X)=A X B+B X A$. If $A$ and $B^{*}$ are injective operators then $E$ is injective.

Proof. If either $A X B=0$ or $B X A=0$ with $A$ injective, then we have that $X B=0=B^{*} X=0$ implies $X^{*}=0=X$ since $B^{*}$ is injective. Thus, $E$ is injective.

Proposition 4.2. Let $A=\left(A_{1}, A_{2}, . . A_{n}\right)$ and $B=\left(B_{1}, B_{2}, . . B_{n}\right)$ with $A_{i}, B_{i}$ be operators in $B(H)$ such that $\sum_{i=1}^{n} A_{i} A_{i}^{*} \leq 1, \sum_{i=1}^{n} A_{i} A_{i}^{*} \leq 1, \sum_{i=1}^{n} B_{i} B_{i}^{*} \leq 1$, and $\sum_{i=1}^{n} B_{i} B_{i}^{*} \leq 1$. If $E$ is the elementary operator defined on $C_{p: 1 \leq p<\infty}$ by $E(X)=\sum_{i=1}^{n} A_{i} X B_{i}-X$ then ker $E=k e r \tilde{E} \Rightarrow$ ker $E^{\dagger}=$ ker $\tilde{E}^{\dagger}$ Moreover, if $E(S)=0=\tilde{E}(S)$ for some compact operator $S$, then $\left[|S|, B_{i}\right]=0 \forall 1 \leq i<n$. 
Proof. We have that $E^{\dagger}(S)=0 \Leftrightarrow \tilde{E} S^{*}=0$. Then from the equality, $\operatorname{Ker} E=\operatorname{Ker} \tilde{E}$. It follows that $E^{\dagger}(S)=0 \Leftrightarrow E\left(S^{*}\right)=0 \Leftrightarrow \tilde{E}^{\dagger}(S)=0$. The rest is trivial.

At this point we give certain necessary conditions and characterization of the operators in $C_{p}$-classes whose kernels are orthogonal to the ranges of certain kinds of elementary operators, in particular, we consider the Jordan elementary operator.

Proposition 4.3. Let $E$ be an elementary operator defined on $C_{p}(H)$ then $\forall S, X \in C_{p}(H), \| E(X)+$ $S\left\|_{p} \geq\right\| S \|_{p} \Rightarrow S \in$ ker $E$ then $E(X)=A X B$ with $A^{*}$ and $B$ injective operators $E(X)=A X B-$ $C X D$, where $A, B$ normal operators, $D, C^{*}$ hyponormal operators with $[A, C]=[B, D]=0$ and $\operatorname{ker} A^{*} \bigcap \operatorname{ker} C^{*}=0=\operatorname{ker} B \bigcap \operatorname{ker} D$.

Proof. The duality adjoint $E^{\dagger}$ is defined by $E^{\dagger}(X)=B X A$ and using a result of [6], we get $E^{\dagger}$ is injective and hence orthogonal. So, the result follows by Proposition 3.1. Next, we have $E^{\dagger}(X)=B X A-D X C$ and applying the result of [25], we get $E^{\dagger}$ is orthogonal, and so by [12], the proof is complete.

Theorem 4.4. Let $A, B \in N A(H)$ be hyponormal operators, such that $A B=B A$, and let $\mathcal{U}(X)=$ $A X B+B X A$. Furthermore, suppose that $A^{*} A+B^{*} B>0$. If $S \in \operatorname{KerU}$, then $|\|\mathcal{U}(X)+S\|| \geq|\|S\||$.

Proof. Follows trivially from the proof of the sum of two basic elementary operators as shown in [9] and from the fact that $|\| \cdot|||$ is a unitarily invariant norm.

We extend Theorem 4.4 to distinct hyponormal operators $A, B, C, D \in N A(H)$ in the theorem below.

Theorem 4.5. Consider $A, B, C, D \in N A(H)$ as hyponormal operators, such that $A C=C A, B D=$ $D B, A A^{*} \leq C C^{*}, B^{*} B \leq D^{*} D$. Let the Jordan elementary operator be give as $\mathcal{U}(X)=A X B+C X D$ and $S \in N A(H)$ satisfying $A S B=C S D$, then $\|\mathcal{U}(X)+S\| \geq\|S\|$, for all $X \in N A(H)$.

Proof. Since $\mathcal{U}(X)=A X B+C X D$ and the fact that $A C=C A, B D=D B, A A^{*} \leq C C^{*}$, $B^{*} B \leq D^{*} D$, then it is easy to see that the operator is injective. So, with $S \in N A(H)$ satisfying $A S B=C S D$, then $\|\mathcal{U}(X)+S\| \geq\|S\|$ follows analogously from the proof of the sum of two basic elementary operators as shown in [18].

Theorem 4.6. Consider $A, B, C, D \in N A(H)$ as hyponormal operators, such that $A C=C A$, $B D=D B, A A^{*} \leq C C^{*}, B^{*} B \leq D^{*} D$. Let the Jordan elementary operator be given as $\mathcal{U}(X)=$ $A X B-C X D$ and $S \in N A(H)$ satisfying $A S B=C S D$, then $\|\mathcal{U}(X)+S\| \geq\|S\|$, for all $X \in$ $N A(H)$. 
Proof. From $A A^{*} \leq C C^{*}$ and $B^{*} B \leq D^{*} D$, let $A=C U$, and $B=V D$, where $U, V$ are unitaries. So we have $A X B-C X D=C U X V D-C X D=C(U X V-X) D$. Assume $C$ and $D^{*}$ are injective, $A S B=C S D$ if and only if $U S V=S$. Moreover, $C$ and $U$ commute. Indeed, from $A=C U$ we obtain $A C=C U C$. Therefore, $C(A-U C)=0$. Thus since $C$ is injective $A=C U$. Similarly, $D$ and $V$ commute. So, $\|\mathcal{U}(X)+S\|=\|[A X B-C X D]+S\|=\|[U(C X D) V-C X D]+S\| \geq$ $\|S\|, \forall X \in N A(H)$. The rest is clear from the analogous assertions in [20] for hyponormal operators.

\section{CONCLUSION}

In this paper, we have given necessary conditions and characterize the elements that are orthogonal to the range of an operator defined in certain classes of normed spaces. We have also given range-kernel orthogonality conditions for elementary operators defined on $C_{p}$-classes and on norm-attainable class, particularly, the Jordan elementary operators.

\section{ACKNOWLEDGEMENTS}

The author is thankful to the referees for their careful reading and valuable contributions to this work.

\section{REFERENCES}

[1] C. Benitez, Orthogonality in Normed Linear Spaces: A Classification of the Different Concepts and Some Open Problems, Rev. Math. 2 (1989), 53-57.

[2] R. Bhatia and P. Semrl, Orthogonality of Matrices and Some Distance Problems, Linear Algebra Appl. 287 (1999), 77-85.

[3] J. Chmielinski, T. Stypula, P. Wójcik, Approximate Orthogonality in Normed Spaces and Its Applications, Linear Algebra Appl. 531 (2017), 305-317.

[4] W. Kangogo, N. B. Okelo and O. Ongati, On Centre Properties of Irreducible Subalgebras of Compact Elementary Operators, Open J. Math. Anal. 4(2) (2020), 80-88.

[5] D. J. Keckic, Orthogonality in $C_{1}$ and $C_{\infty}$ Spaces and Normal Derivations, J. Oper. Theory 51 (2004), 89-104.

[6] D. J. Keckic, Orthogonality of the Range and Kernel of Some Elementary Operators, Proc. Amer. Math. Soc. 11 (2008), 3369-3377.

[7] S. Mecheri, On the Range and Kernel of the Elementary Operators $\sum_{i=1}^{n} A_{i} X B_{i}-X$, Acta Math. Univ. Comnianae, 52 (2003), 119-126.

[8] N. B. Okelo, On Norm-Attainable Functionals in Banach Spaces, Asia Pac. J. Math, 7 (2020), 27.

[9] N. B. Okelo, Characterization of Absolutely Norm Attaining Compact Hyponormal Operators, Proc. Int. Math. Sci. 2(2) (2020), 96-102. 
[10] N. B. Okelo, On Properties of Inner Product Type Integral Transformers, Open J. Math. Anal. 4(2) (2020), 160-169.

[11] N. B. Okelo, Fixed Points Approximation for Nonexpansive Operators in Hilbert Spaces, Int. J. Open Probl. Comput. Math. 14(1) (2021), 1-5.

[12] N. B. Okelo, P. O. Mogotu, On Norm Inequalities and Orthogonality of Commutators of Derivations, Italian J. Pure Appl. Math. 44 (2020), 163-169.

[13] N. B. Okelo, Norms of Self-Adjoint Two-Sided Multiplication Operators in Norm-Attainable Classes, MATLAB J. 6 (2020), 77-79.

[14] N. B. Okelo, On Convex Optimization in Hilbert Spaces, Maltepe J. Math. 2 (2019), 89-95.

[15] N. B. Okelo, J. O. Agure and D. O. Ambogo, Norms of Elementary Operators and Characterization of Norm-Attainable Operators, Int. J. Math. Anal. 24 (2010), 1197-1204.

[16] N. B. Okelo, The Norm-Attainability of Some Elementary Operators, Appl. Math. E-Notes 13 (2013), 1-7.

[17] N. B. Okelo, $\alpha$-Supraposinormality of Operators in Dense Norm-Attainable Classes, Univ. J. Math. Appl. 2 (2019), 42-43.

[18] N. B. Okelo, On Orthogonality of Elementary Operators in Norm-Attainable Classes, Taiwan. J. Math. 24 (2020), 119-130.

[19] N. B. Okelo, M. O. Okongoand, S. A. Nyakiti, On Projective Tensor Norm and Norm-Attainable $\alpha$-Derivations, Int. J. Contemp. Math. Sci. 5 (2010), 1969-1975.

[20] N. B. Okelo, J. O. Agure and P. O. Oleche, Various Notions of Orthogonality in Normed Spaces, Acta Mathematica Scientia, 33(5) (2013), 1387-1397.

[21] Okelo N. B., On Norm-Attainable Operators in Banach Spaces, J. Funct. Spaces, 1 (2020), 1-6.

[22] N. B. Okelo, On Certain Conditions for Convex Optimization in Hilbert Spaces, Khayyam J. Math. 5 (2019), 108-112.

[23] N. B. Okelo, J. O. Agure and D. O. Ambogo, Norms of Elementary Operators and Characterization of Norm - Attainable Operators, Int. J. Math. Anal. 24 (2010), 687-693.

[24] K. Paul, D. Sain, A. Mal, Approximate Birkhoff-James Orthogonality in the Space of Bounded Linear Operators, Linear Algebra Appl. 537 (2018), 348-357.

[25] A. Turnsek, Orthogonality in $\mathcal{C}_{p}$-Classes, Monatsh. Math. 132 (2001), 349-354. 